\title{
Gene-environment interactions determine risk for dementia: the influence of lifestyle on genetic risk for dementia
}

\author{
Kelley T. Patten, Pamela J. Lein \\ Department of Molecular Biosciences, University of California at Davis, Davis, CA, USA \\ Correspondence to: Pamela J. Lein. 2009 VM3B, 1089 Veterinary Medicine Drive, Davis, CA 95616, USA. Email: pjlein@ucdavis.edu. \\ Provenance: This is an invited article commissioned by the Section Editor Dr. Can Sheng (Department of Neurology, the First Hospital of Tsinghua \\ University, Beijing, China). \\ Comment on: Lourida I, Hannon E, Littlejohns TJ, et al. Association of Lifestyle and Genetic Risk With Incidence of Dementia. JAMA 2019. [Epub \\ ahead of print].
}

Submitted Sep 11, 2019. Accepted for publication Sep 26, 2019.

doi: 10.21037/atm.2019.09.155

View this article at: http://dx.doi.org/10.21037/atm.2019.09.155

Modifiable lifestyle factors such as diet, exercise, alcohol intake, and smoking, have well-established associations with risk of dementia. A poor diet, insufficient exercise, excessive alcohol intake, and smoking are individually associated with increased risk for dementia, while adhering to a healthy diet, abstaining from smoking, exercising regularly, and consuming alcohol in moderation have all been associated with decreased risk. However, there is a paucity of research regarding the cumulative impact of these factors on dementia risk. Moreover, studies of lifestyle typically have not taken into account an individual's overall genetic predisposition for dementia. This is a significant confound given that genetic background is thought to play a predominant role in much of dementia. Large genomewide association studies (GWAS) have made substantial progress in unearthing susceptibility loci for dementia, but most of these have not been examined in conjunction with lifestyle $(1,2)$. Thus, prior to the recent $7 A M A$ study by Lourida et al. (3), the extent to which overall lifestyle can offset or exacerbate an individual's genetic risk for dementia had been examined only in small cohorts, and/or in the context of specific genetic risk factors (i.e., $A P O E-\varepsilon 4$ ). This study is the first to examine the association between lifestyle and dementia using a composite measure of lifestyle and a polygenic measure of genetic risk for dementia. Consequently, it provides strong evidence that modifiable, external factors such as lifestyle can influence dementia risk, regardless of genetic background.

This retrospective study measured incident dementia in 196,383 participants from the United Kingdom. Mean age of individuals at enrollment was 65.8 years for those who developed dementia $(n=1,769)$, and 64.1 years for those who did not $(\mathrm{n}=194,614)$. Participants, who did not have prevalent dementia at study enrollment, were followed for approximately 8 years or until one of the following criteria was met: (I) diagnosis of dementia, (II) death, or (III) loss to follow-up. The authors generated a polygenic risk score based on single nucleotide polymorphisms (SNPs) previously identified in a GWAS (2), ultimately including over 200,000 SNPs associated with Alzheimer's disease (AD). For each individual, the number of risk alleles per SNP was weighted by the strength of that SNP's association with $\mathrm{AD}$, and these were summed to generate an overall risk score. Individuals were then divided into either low, intermediate, or high genetic risk categories.

Individuals were also grouped into either favorable, intermediate, or unfavorable lifestyles, based on selfreported responses to a questionnaire at enrollment on smoking status, physical activity level, diet, and alcohol consumption. One significant limitation to this approach is that lifestyle habits measured at enrollment do not necessarily reflect lifestyle habits throughout life. Since dementia pathogenesis is thought to initiate years or even decades prior to manifestation of symptoms, lifestyle factors in mid-to-early life are arguably more or equally important to those later in life. A long-term prospective cohort study that carefully documents lifestyle habits throughout life and measures incident dementia would be needed to address this issue. Consequently, published prospective cohort studies of incident dementia and lifestyle factors have been restricted 
to small sample sizes with limited statistical power. While retrospective studies can be an effective way to circumvent this barrier, it will be important for future studies that enroll elderly patients to establish how well lifestyle metrics ascertained at study entry reflect the lifestyle habits of previous decades. Similarly, preclinical studies using validated animal models of dementia may be useful in identifying therapeutic window(s) during which lifestyle factors effectively influence dementia risk.

The key findings of the Lourida et al. study are: (I) an unhealthy lifestyle increases risk for dementia, independent of genetic risk; and (II) a healthy lifestyle can decrease risk for dementia, independent of genetic risk. However, it is important to note that for individuals with a high genetic risk score, following a favorable lifestyle may decrease risk of incident dementia, but it does not completely eliminate their risk for developing dementia. As noted by the authors, in individuals with high genetic risk of dementia, the difference between following a favorable vs. unfavorable lifestyle roughly translates to prevention of dementia in $1 / 121$ individuals over a 10 -year period. Whether lifestyle habits also affected the severity or time-to-onset of dementia was not specifically addressed. Considering that this study found that the genetic contribution to dementia risk was decreased, but not eliminated by a healthy lifestyle, it is worth exploring whether therapeutics can amplify the effects of a favorable lifestyle in genetically susceptible individuals.

Given the challenges of conducting a randomized controlled trial (RCT) to evaluate whether lifestyle factors, such as diet and exercise, modify the genetic risk of AD and other dementias, preclinical studies will be critical for generating data regarding the value of various lifestyle factors in influencing genetic risk. To that end, here we comment on recent preclinical and clinical studies of various non-drug interventions, and discuss their potential to be used as therapeutics, or in conjunction with lifestyle changes to mitigate dementia.

\section{Diet}

Ketogenic diets and caloric restriction have both been reported to have beneficial effects on cognitive function. These approaches may reflect overlapping mechanisms, since caloric restriction effectively increases ketone bodies (4). Ketones and a ketogenic diet have well-established beneficial effects in the treatment and control of epilepsy; however, their benefit(s) in $\mathrm{AD}$ and age-related cognitive decline is an emerging area of research (5). In rodents, ketogenic studies are typically performed using exogenous ketones, and several studies have demonstrated improved cognitive function and decreased $\beta$-amyloid load in $\mathrm{AD}$ transgenic mice administered ketones $(6,7)$. Similarly, a small clinical trial in humans with mild cognitive impairment (MCI) showed that a low-carbohydrate (ketogenic) diet improved verbal memory (8). While these data are promising, there are critical challenges associated with leveraging the beneficial effects of ketones as a therapeutic strategy for treating dementia. These include issues of patient compliance due to poor tolerability of longterm ketogenic diets, although this may be improved by using a different type of ketone body $(9,10)$. Perhaps more problematic is that long-term ketogenic diets and prolonged fasting may lead to or exacerbate nutritional deficits in the elderly (5). Therefore, it will be important to establish the efficacy of ketone supplementation prior to and during the symptomatic period.

\section{Probiotic supplementation}

Altered gut microbiota have been observed in multiple neurological conditions including dementia and $\mathrm{AD}$, which has spurred exploration of the microbiome as a therapeutic target (11). Indeed, recent clinical trials of prebiotic or probiotic supplementation to change the microbiome have shown promising results in $\mathrm{AD}$ or MCI patients, even with short-term treatment (12-15). For example, one small exploratory study found that a 4-week probiotic treatment resulted in improvements in the Mini-Mental State Examination for cognitive impairment (14), though these results need to be confirmed in a RCT. Moreover, though clinical and preclinical studies agree that there is a general decrease in microbial diversity during $\mathrm{AD}(16,17)$, these changes comprise many bacterial species, and probiotic supplementation has not been exhaustively interrogated for all of these. Probiotic supplementation studies typically use Lactobacillus and Bifidobacterium species, as these are thought to decrease during $\mathrm{AD}$, and are general well-tolerated by patients. However, it is unclear whether other species or more targeted supplementation would have similar beneficial effects. More importantly, it is not clear how probiotics are exerting their effects on dementia or MCI. Gut microbiota have been shown to produce neurotransmitters and peptides involved in blood-brain barrier stability, to alter the immune response, and to increase insulin resistance (11). While all of these pathways have been implicated in $\mathrm{AD}$, 
whether the effects of probiotic supplementation on the microbiome composition of the gut influences $\mathrm{AD}$ specifically through one of these pathways has yet to be shown. However, one intriguing finding from these studies is that peripheral immune markers of inflammation are often reduced with probiotic administration, suggesting that perhaps probiotics are mediating their effects on the brain via immune pathways. Consistent with these findings from human studies, probiotic administration to $\mathrm{AD}$-transgenic mice reduces oxidative stress and improves $\mathrm{AD}$ phenotypes in the brain $(18,19)$.

Overall, probiotics are particularly intriguing as a therapeutic, as they are generally well-tolerated and can be easily administered. However, as with a ketogenic diet, further studies are needed to determine the dose and length of treatment that provide optimized benefits while ensuring tolerability in dementia patients.

\section{Exercise}

Exercise is perhaps the lifestyle factor most consistently associated with reduced risk of dementia, and studies have found that the positive effect(s) of exercise hold whether physical activity occurs in midlife, well in advance of dementia symptoms (20), or whether it occurs in elderly patients already diagnosed with dementia (21). Though numerous studies have documented the benefits of exercise in ameliorating dementia, it is important to note that the mechanism by which this occurs differ with age. For example, exercise in midlife may have long-term cardiovascular benefits that ultimately reduce dementia risk (22), while exercise in dementia patients may increase neurotrophic growth factors, or ameliorate depressive symptoms $(23,24)$.

\section{The path forward}

As Lourida et al. discuss, SNPs associated with AD affect various biological processes including immune function, lipid homeostasis, endocytic processing, and cholesterol transport. Thus, it is possible that stratifying individuals by genetic variant function may reveal more pronounced effects of certain lifestyle factors in mitigating genetic risk. For example, risk variants in TREM2 and $H L A-D R B 5-D B R 1$ are known to influence microglial function and inflammatory processes. Would probiotics that modulate inflammation be most efficacious in these individuals? APOE- $\varepsilon 4$ is thought to play a role in cholesterol homeostasis and lipid transport.
Would $A P O E-\varepsilon 4$ individuals benefit most from a longterm ketogenic diet? Alternatively, perhaps the benefits of a healthy lifestyle are greater than the sum of its parts. Admittedly, the biological pathways implicated in $\mathrm{AD}$ and age-related dementias often interact with one another, and many $\mathrm{AD}$ risk genes have pleiotropic effects. Over a lifetime, these interactive effects are likely to be magnified, and thus, a single therapeutic targeting a particular pathway (i.e., cholesterol transport) may be ineffective, or only effective when the intervention is administered early in life. Overall, future large-scale cohort studies that stratify by genetic variant function will be important in determining whether particular lifestyle factors should be targeted to specific individuals. Likewise, preclinical studies will be essential for determining the therapeutic window for effective lifestyle interventions, and for determining whether the effects of specific genetic variants on $\mathrm{AD}$ can be uniquely targeted by particular lifestyle interventions.

\section{Acknowledgments}

None.

\section{Footnote}

Conflicts of Interest: The authors have no conflicts of interest to declare.

Ethical Statement: The authors are accountable for all aspects of the work in ensuring that questions related to the accuracy or integrity of any part of the work are appropriately investigated and resolved.

\section{References}

1. Kunkle BW, Grenier-Boley B, Sims R, et al. Genetic meta-analysis of diagnosed Alzheimer's disease identifies new risk loci and implicates Abeta, tau, immunity and lipid processing. Nat Genet 2019;51:414-30.

2. Lambert JC, Ibrahim-Verbaas CA, Harold D, et al. Meta-analysis of 74,046 individuals identifies 11 new susceptibility loci for Alzheimer's disease. Nat Genet 2013;45:1452-8.

3. Lourida I, Hannon E, Littlejohns TJ, et al. Association of Lifestyle and Genetic Risk With Incidence of Dementia. JAMA 2019. [Epub ahead of print].

4. Lin AL, Zhang W, Gao X, et al. Caloric restriction increases ketone bodies metabolism and preserves blood 
flow in aging brain. Neurobiol Aging 2015;36:2296-303.

5. Taylor MK, Swerdlow RH, Sullivan DK. Dietary Neuroketotherapeutics for Alzheimer's Disease: An Evidence Update and the Potential Role for Diet Quality. Nutrients 2019. doi: 10.3390/nu11081910.

6. Kashiwaya Y, Bergman C, Lee JH, et al. A ketone ester diet exhibits anxiolytic and cognition-sparing properties, and lessens amyloid and tau pathologies in a mouse model of Alzheimer's disease. Neurobiol Aging 2013;34:1530-9.

7. Yin JX, Maalouf M, Han P, et al. Ketones block amyloid entry and improve cognition in an Alzheimer's model. Neurobiol Aging 2016;39:25-37.

8. Krikorian R, Shidler MD, Dangelo K, et al. Dietary ketosis enhances memory in mild cognitive impairment. Neurobiol Aging 2012;33:425.e19-27.

9. Taylor MK, Sullivan DK, Mahnken JD, et al. Feasibility and efficacy data from a ketogenic diet intervention in Alzheimer's disease. Alzheimers Dement (N Y) 2017;4:28-36.

10. Augustin K, Khabbush A, Williams S, et al. Mechanisms of action for the medium-chain triglyceride ketogenic diet in neurological and metabolic disorders. The Lancet Neurology 2018;17:84-93.

11. Pistollato F, Sumalla Cano S, Elio I, et al. Role of gut microbiota and nutrients in amyloid formation and pathogenesis of Alzheimer disease. Nutrition Reviews 2016;74:624-34.

12. Agahi A, Hamidi GA, Daneshvar R, et al. Does Severity of Alzheimer's Disease Contribute to Its Responsiveness to Modifying Gut Microbiota? A Double Blind Clinical Trial. Front Neurol 2018;9:662.

13. Bonfili L, Cecarini V, Berardi S, et al. Microbiota modulation counteracts Alzheimer's disease progression influencing neuronal proteolysis and gut hormones plasma levels. Sci Rep 2017;7:2426.

14. Leblhuber F, Steiner K, Schuetz B, et al. Probiotic Supplementation in Patients with Alzheimer's Dementia - An Explorative Intervention Study. Curr Alzheimer Res 2018;15:1106-13.

15. Akbari E, Asemi Z, Daneshvar Kakhaki R, et al. Effect of
Probiotic Supplementation on Cognitive Function and Metabolic Status in Alzheimer's Disease: A Randomized, Double-Blind and Controlled Trial. Front Aging Neurosci 2016;8:256.

16. Vogt NM, Kerby RL, Dill-McFarland KA, et al. Gut microbiome alterations in Alzheimer's disease. Sci Rep 2017;7:13537.

17. Mancuso C, Santangelo R. Alzheimer's disease and gut microbiota modifications: The long way between preclinical studies and clinical evidence. Pharmacol Res 2018;129:329-36.

18. Abraham D, Feher J, Scuderi GL, et al. Exercise and probiotics attenuate the development of Alzheimer's disease in transgenic mice: Role of microbiome. Exp Gerontol 2019;115:122-31.

19. Bonfili L, Cecarini V, Cuccioloni M, et al. SLAB51 Probiotic Formulation Activates SIRT1 Pathway Promoting Antioxidant and Neuroprotective Effects in an AD Mouse Model. Mol Neurobiol 2018;55:7987-8000.

20. Andel R, Crowe M, Pedersen NL, et al. Physical Exercise at Midlife and Risk of Dementia Three Decades Later: A Population-Based Study of Swedish Twins. J Gerontol A Biol Sci Med Sci 2008;63:62-6.

21. Heyn P, Abreu BC, Ottenbacher KJ. The effects of exercise training on elderly persons with cognitive impairment and dementia: a meta-analysis. Arch Phys Med Rehabil 2004;85:1694-704.

22. Rovio S, Kåreholt I, Helkala EL, et al. Leisure-time physical activity at midlife and the risk of dementia and Alzheimer's disease. Lancet Neurol 2005;4:705-11.

23. Coelho FG, Vital TM, Stein AM, et al. Acute aerobic exercise increases brain-derived neurotrophic factor levels in elderly with Alzheimer's disease. J Alzheimers Dis 2014;39:401-8.

24. Pereira DS, de Queiroz BZ, Miranda AS, et al. Effects of physical exercise on plasma levels of brain-derived neurotrophic factor and depressive symptoms in elderly women--a randomized clinical trial. Arch Phys Med Rehabil 2013;94:1443-50.
Cite this article as: Patten KT, Lein PJ. Gene-environment interactions determine risk for dementia: the influence of lifestyle on genetic risk for dementia. Ann Transl Med 2019;7(Suppl 8):S322. doi: 10.21037/atm.2019.09.155 